\title{
INFLUÊNCIA DO ESPAÇAMENTO DE PLANTIO NA PRODUÇÃO ENERGÉTICA DE CLONES DE Corymbia e Eucalyptus
}

\author{
Emerson Delano Lopes ${ }^{1}$, Marcelo Luiz de Laia ${ }^{1 *}$, Alexandre Soares Santos ${ }^{1}$, Guilherme Mendes Soares ${ }^{1}$, \\ Ricardo Wagner Pinto Leite ${ }^{2}$, Nivaldo de Souza Martins ${ }^{2}$ \\ ${ }^{1}$ Universidade Federal dos Vales do Jequitinhonha e Mucuri, Diamantina, Minas Gerais, Brasil - emerson.lopes@ifnmg.edu.br; \\ marcelo.laia@ufvjm.edu.br; alexandreletam@gmail.com; guilhermemsoares@yahoo.com.br \\ ${ }^{2}$ Aperam Bioenergia S/A, Itamarandiba, Minas Gerais, Brasil - ricardo-wagner.leite@ aperam.com; nivaldo.martins@aperam.com
}

Recebido para publicação: 14/06/2016 - Aceito para publicação: 05/01/2017

\begin{abstract}
Resumo
O presente trabalho teve como objetivo avaliar o crescimento inicial, a massa específica básica, a produção da biomassa e analisar quimicamente a madeira e casca de dois clones de Eucalyptus (um híbrido espontâneo de Eucalyptus urophylla S.T. Blake; um híbrido tri-cross de Eucalyptus urophylla S.T. Blake x (E. camaldulensis Dehn x E. grandis Hill ex Maiden)) e um híbrido de Corymbia citriodora (Hook) K. D. Hill \& L. A. Johnson x C. torelliana (F. Muell) K. D. Hill \& L. A. Johnson, distribuídos em três espaçamentos de plantio: $3 \times 3 \mathrm{~m}$; 3x1,5 m; e 3x1 m, na idade de 12 meses. O estudo foi conduzido num experimento localizado no município de Itamarandiba, MG, em delineamento experimental inteiramente casualizado com quatro repetições, em um esquema fatorial $3 \times 3$, considerando como níveis dos fatores os diferentes clones e os espaçamentos de plantio. Observou-se para os três clones, que o espaçamento $3 \times 3 \mathrm{~m}$ proporcionou plantas com maior crescimento em diâmetro à altura do peito (DAP) em relação aos demais espaçamentos. Os diferentes espaçamentos de plantio não induziram a variação da massa específica básica da madeira e das cascas nos clones estudados. O aumento da densidade de plantio apresentou relação direta com a produção de biomassa por unidade de área. Observou-se decréscimo dos teores de lignina na madeira, para os dois clones de Eucalyptus, do maior espaçamento para os espaçamentos mais adensados.
\end{abstract}

Palavras-chave: Floresta energética; Biomassa; Biocombustível.

\begin{abstract}
Evaluation Corymbia and Eucalyptus clones under different spacings order to produce bioenergy. This study aimed to evaluate the initial growth, the basic density, the production of biomass and chemically analyzing of the wood and bark of two Eucalyptus clones (a hybrid of Eucalyptus urophylla S.T. Blake, a hybrid tri-cross Eucalyptus urophylla S.T. Blake x (E. camaldulensis Dehn x E. grandis Hill ex Maiden)) and a hybrid Corymbia citriodora (Hook) K. D. Hill \& L. A. Johnson x C. torelliana (F. Muell) K. D. Hill \& L. A. Johnson distributed in three planting spacings: $3 \times 3 \mathrm{~m}, 3 \times 1,5 \mathrm{~m}, 3 \times 1 \mathrm{~m}$, at the age of 12 months. The study was conducted in an experiment located in the county Itamarandiba, MG, in a completely randomized design with four replications, in a factorial $3 \times 3$, considering how levels of the factors the different clones and planting spacings. It was observed for the three clones that the $3 \times 3 \mathrm{~m}$ spacing provided plants with greater growth in diameter at breast height (DAP) in relation to the other spacings. The different planting spacings did not induce the variation of the basic specific mass of the wood and the bark in the studied clones. The increase in planting density had a direct relation with biomass production per unit area. There was a decrease in lignin contents in the wood for two clones of Eucalyptus, the larger spacing for the denser spacings.

Keywords: Energy forest; Biomass; Biofuel.
\end{abstract}

\section{INTRODUÇÃO}

A forte pressão exercida pela sociedade para a redução da emissão de poluentes impõe a uma maior busca por biocombustíveis provenientes de fontes renováveis, e nesse cenário, destaca-se a biomassa florestal (CARNEIRO et al., 2014).

As espécies do gênero Eucalyptus e Corymbia se destacam entre as diversas opções para o fornecimento de biomassa florestal em razão do seu rápido crescimento, alta massa energética, tolerância a condições extremas, e possibilidade de adensamento dos plantios. A eucaliptocultura, nomenclatura utilizada para designar o cultivo de espécies dos gêneros Eucalyptus e Corymbia, no país é uma das mais avançadas do mundo em razão dos ganhos proporcionados pelo melhoramento genético das espécies que compõem estes gêneros e pelas 
modernas tecnologias de implantação e de condução dos povoamentos. A utilização da madeira desses gêneros já é consagrada para o uso energético, tendo grande importância nos setores comercial, industrial e residencial.

Apoiado nas inovações do setor florestal brasileiro, como os avanços do melhoramento genético florestal, ressurge o conceito de florestas energéticas que teriam como vocação o suprimento da demanda da biomassa energética, preferencialmente, em rotações mais curtas. Tais florestas objetivam a produção de matériaprima em quantidade, qualidade, e num intervalo de tempo reduzido, quando comparado aos sistemas silviculturais convencionais (GUERRA et al., 2012).

O potencial energético de uma floresta sofre influência de fatores como a constituição genética da espécie, tratamentos silviculturais, idade, local de plantio e as interações dos diversos fatores, o que pode afetar diretamente na qualidade e no uso final da madeira (PROTÁSIO et al., 2014; MOULIN et al., 2015). Um dos principais fatores envolvidos no manejo das florestas energéticas é a correta definição do espaçamento de plantio. O espaçamento de plantio é determinado pela distância entre linhas e entre plantas, e têm sido amplamente estudado para a obtenção das respostas quanto ao material genético mais apropriado a cada sítio florestal, pois esses podem responder diferentemente à redução ou ampliação do espaçamento (FERREIRA et $a l ., 2014)$. Outro fator importante na formação das florestas energéticas é a correta seleção de clones superiores. Segundo Botrel et al. (2010), a seleção de clones oriundos dos programas de melhoramento florestal sempre foi baseada nos valores fenotípicos das características dendrométricas, como o DAP, altura e volume, entretanto, isso não garantiu a seleção de genótipos com características tecnológicas desejadas para usos específicos. Nesse sentido, estudos sobre a qualidade da madeira também devem ser conduzidos, haja vista a sua importância para a correta seleção de materiais genéticos aptos para o uso energético.

A massa específica básica é um parâmetro que apresenta alta eficiência para expressar a qualidade da madeira, a fim de prognosticar sua variação em diferentes usos, em razão da íntima relação com outras várias propriedades (WASHUSEN et al., 2005). A massa específica básica repercute diretamente na produção da biomassa seca do individuo, e tal característica deve influenciar na seleção dos materiais genéticos com melhores taxas de conversão energética (TRUGILHO et al., 2010). Tal parâmetro é representado pela concentração de massa por volume e, em razão disso, influencia a velocidade da queima durante a produção direta de energia (ELOY et al., 2014).

A composição química dos materiais lenhosos, como os teores de lignina e de holocelulose (celulose e hemicelulose), exercem grande influência no processo de beneficiamento do produto florestal, e o teor de cada constituinte é influenciado pela condição ambiental (ZANUNCIO et al., 2013). Assim, estudos das propriedades silviculturais e da biomassa das espécies florestais são fundamentais para a seleção de materiais genéticos que produzam biomassa energética em quantidade e na qualidade desejada.

Dentro desse contexto, o presente trabalho teve como objetivo avaliar a qualidade e a produção da biomassa de clones híbridos de Eucalyptus e Corymbia conduzidos em diferentes espaçamentos de plantio, aos 12 meses de idade.

\section{MATERIAL E MÉTODOS}

\section{Caracterização da área experimental}

$\mathrm{O}$ experimento foi implantado em propriedade da empresa Aperam Bioenergia S/A localizada no município de Itamarandiba, Minas Gerais. A região experimental localiza-se nas coordenadas geográficas a $17^{\circ} 44^{\prime} 22^{\prime}$ S S de latitude e $42^{\circ} 54^{\prime} 12^{\prime}$ 'W de longitude e altitude de $900 \mathrm{~m}$. O clima da região é definido como tropical de altitude, com duas estações bem definidas. A temperatura média da região é de $21,2^{\circ} \mathrm{C}$, a umidade relativa do ar média anual varia entre 60 a 70\%, e o índice pluviométrico médio anual é de $1.132 \mathrm{~mm}$. O solo da região é classificado como argissolo amarelo típico textura arenosa/média fase relevo plano, e a tipologia florestal predominante é o cerrado.

\section{Material Vegetal}

Foram utilizados três clones da empresa Aperam Bioenergia, sendo um híbrido espontâneo de Eucalyptus urophylla (Clone 1), um híbrido tri-cross de Eucalyptus urophylla x (E. camaldulensis x E. grandis) (Clone 2), e um híbrido de Corymbia citriodora x $C$. torelliana (Clone 3 ), que foram implantados em três diferentes espaçamentos de plantio: $3 \times 3 \mathrm{~m}$ (9 m²/planta), 3x1,5 m (4,5 m²/planta), e 3x1 m (3 m²/planta).

\section{Seleção, medição, determinação dos volumes e preparo das amostras}

Aos 12 meses de idade, selecionaram-se quatro árvores de diâmetro médio para cada clone, nos três espaçamentos de plantio, totalizando 36 amostras, que foram medidas quanto à altura total (Ht) e o diâmetro a altura do peito (DAP). Com a finalidade de determinar o volume sólido $\left(\mathrm{m}^{3}\right)$ individual, as 36 árvores foram abatidas e cubadas rigorosamente utilizando-se o método de Smalian, considerando os diâmetros com casca. 
Os incrementos médios anuais de madeira ou de casca foram obtidos pela multiplicação do volume médio individual das árvores amostras de cada clone, obtidos pela cubagem rigorosa, em função das densidades de plantio: $3 \times 3 \mathrm{~m}$ (1111 plantas.ha $\left.{ }^{-1}\right)$, 3x1,5 m (2222 plantas.ha $\left.^{-1}\right)$, e 3x1 m (3333 plantas.ha $\left.{ }^{-1}\right)$.

Após a cubagem rigorosa das árvores retiraram-se discos, com as cascas, de $2,5 \mathrm{~cm}$ de espessura nas posições $0 \%, 25 \%, 50 \%, 75 \%$ da altura total de cada árvore abatida, além de um disco extra a 1,30 m de altura do solo (DAP). As cascas dos discos foram medidas com auxílio de paquímetro, para determinar o percentual de casca.

\section{Análise química da madeira e casca}

Para a análise química da madeira e das cascas dos clones, realizou-se uma amostragem composta dos discos obtidos ao longo do fuste das árvores amostradas. As amostras de madeira e casca foram trituradas em moinho tipo Willey, para redução da dimensão, e peneiradas, sendo utilizado para as avaliações o material que passou por peneira de malha igual a $2 \mathrm{~mm}$. A caracterização química das amostras foi realizada, em triplicata, através da determinação dos teores de cinzas totais, de acordo com as normas do Instituto Adolph Lutz (IAL, 2008), e de celulose, hemicelulose e lignina pelo método de Van Soest (1970).

\section{Determinação da massa específica básica, biomassa seca, massa de lignina e massa de holocelulose}

Os discos de madeira e as cascas referentes às alturas amostradas foram identificados e destinados à determinação da massa específica básica da madeira e da casca de acordo com a NBR 11941 (ABNT, 2003). A massa específica básica $(\rho b)$ foi considerada como sendo a média aritmética dos pontos de amostragem longitudinal no tronco das árvores, sem considerar a posição do DAP. Para o cálculo da massa específica básica da madeira e casca foi utilizada a equação (1):

$$
\rho b=M_{s} / V_{v(1)}
$$

em que: $\rho b$ : massa específica básica; $V_{v}$ : volume dos corpos-de-prova verdes; e $M_{s}$ : massa dos corpos-de-prova absolutamente secos.

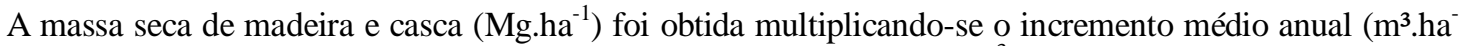
${ }^{1}$.ano $\left.{ }^{-1}\right)$ da madeira e casca pela massa específica básica da madeira e casca $\left(\mathrm{kg} \cdot \mathrm{m}^{-3}\right)$, conforme a equação (2):

$$
M S=I M A \times \rho b_{(2)}
$$

em que: $M S$ : massa seca de madeira ou de casca; IMA: incremento médio anual da madeira e casca; $\rho b$ : massa específica básica da madeira e casca.

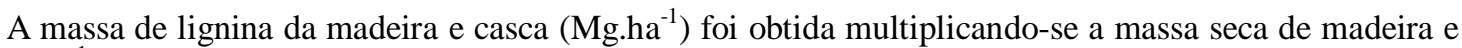

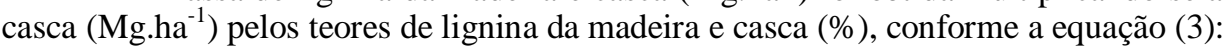

$$
M_{\text {Lig }}=\operatorname{MS} \times \operatorname{Lig}(\%)_{(3)}
$$

em que: MLig: massa lignina; $M S$ : massa seca de madeira e casca; $\operatorname{Lig}(\%)$ : teor de lignina da madeira e casca.

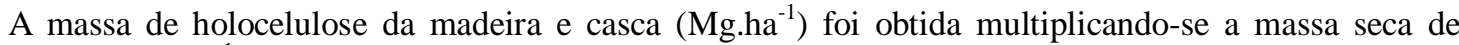
madeira e casca $\left(\mathrm{Mg} \cdot \mathrm{ha}^{-1}\right.$ ) pelos teores de holocelulose da madeira e casca (\%), conforme a equação (4):

$$
M_{\text {Holo }}=\operatorname{MS} \times \operatorname{Holo}(\%)(4)
$$

em que: MHolo: massa holocelulose; MS: massa seca de madeira e casca; $H o l o(\%)$ : teor de holocelulose da madeira e casca.

Para avaliar as características dendrométricas e químicas, a massa específica básica, e as estimativas de massa seca da madeira, da casca, de holocelulose e de lignina, instalou-se um esquema fatorial no delineamento inteiramente casualizado, em que foram comparados três clones em três espaçamentos de plantio, com quatro repetições (árvores), totalizando nove tratamentos e 36 observações. Os dados foram submetidos aos testes de Lilliefors para testar a normalidade e de Cochran para testar a homogeneidade das variâncias e, em seguida, submetidos à análise de variância (ANOVA). Estabelecidas às diferenças significativas, foram feitos os devidos desdobramentos, sendo os tratamentos comparados entre si, por meio do teste de Tukey a 5\% de probabilidade.

\section{RESULTADOS}

Observou-se que a interação clone $\mathrm{x}$ espaçamento foi significativa $(\mathrm{p}<0,05)$ para as variáveis dendrométricas de altura total (Ht), diâmetro à altura do peito (DAP) e incremento médio anual (IMA), assim como 
para os valores de massa seca da madeira, casca e total (MSM, MSC e MST), evidenciando a existência de dependência entre os fatores considerados (Tabela 1). Dessa forma, procedeu-se o seu desdobramento e avaliação do efeito de clone dentro de espaçamento e vice-versa (Tabela 3). Já para a massa específica básica da madeira e casca $\left(\rho b_{\text {madeira e }} \rho b_{\text {casca }}\right.$ ) e para o percentual de casca (\% casca) constatou-se efeito significativo apenas de clone e espaçamento, respectivamente. Para essas variáveis procedeu-se a análise do efeito simples (Figura 1 e Tabela 4).

Tabela 1. Resumo da análise de variância realizada para a altura total (Ht), diâmetro a altura do peito (DAP), incremento médio anual (IMA), percentual de casca (\% casca), biomassa seca da madeira (MSM), biomassa seca da casca (MSC), biomassa seca total (MST), massa especifica básica da casca $\left(\rho b_{\text {casca }}\right)$ e massa especifica básica da madeira ( $\left.\rho b_{\text {madeira }}\right)$.

Table 1. Summary of the analysis of variance performed for total height (Ht), diameter at breast height (DAP), mean annual increment (IMA), percentage of bark (\% casca), dry biomass of wood (MSM), dry bark biomass (MSC), total dry biomass (MST), basic specific mass of the bark $\left(\rho b_{\text {casca }}\right)$ and basic specific mass of the wood ( $\left.\rho b_{\text {madeira }}\right)$.

\begin{tabular}{|c|c|c|c|c|c|c|c|c|c|c|}
\hline \multirow[b]{2}{*}{ Fonte de variação } & \multirow[b]{2}{*}{ GL } & \multicolumn{9}{|c|}{ Quadrado médio } \\
\hline & & $\mathbf{H t}$ & DAP & IMA & $\begin{array}{c}\% \\
\text { casca }\end{array}$ & MSM & MSC & MST & $\rho \mathbf{b}_{\text {casca }}$ & $\rho b_{\text {madeira }}$ \\
\hline Clone & 2 & $8,172 *$ & $8,491 *$ & $232,644 *$ & $7,475^{\mathrm{ns}}$ & $14,891 *$ & $0,611 *$ & $21,393^{*}$ & $11815,6^{*}$ & $51060,1 *$ \\
\hline Espaçamento & 2 & $0,220^{\mathrm{ns}}$ & $2,932 *$ & $161,633 *$ & $21,917^{*}$ & $20,768^{*}$ & $0,382 *$ & $26,788^{*}$ & $799,1^{\mathrm{ns}}$ & $321,6^{\mathrm{ns}}$ \\
\hline Clone*Espaçamento & 4 & $0,447 *$ & $0,409 *$ & $5,564 *$ & $5,077^{\mathrm{ns}}$ & $0,658 *$ & $0,0364 *$ & $0,986^{*}$ & $249,3^{\mathrm{ns}}$ & $405,3^{\mathrm{ns}}$ \\
\hline Erro & 27 & 0,082 & 0,013 & 1,454 & 2,193 & 0,209 & 0,007 & 0,278 & 135,7 & 240,9 \\
\hline Total & 35 & & & & & & & & & \\
\hline
\end{tabular}

GL: graus de liberdade; *significativo ao nível de $5 \%$ de probabilidade ( $\mathrm{p}<0,05)$; ${ }^{\text {ns }}$ não significativo ao nível de $5 \%$ de probabilidade (p $\geq 0,05$ ).

O resumo da análise de variância (ANOVA) para os teores de celulose da madeira e casca, lignina da madeira, biomassa de holocelulose da madeira e casca $\left(\mathbf{M}_{\text {holomad }}, \mathbf{M}_{\text {holocasca }}\right.$ ) e biomassa de lignina da madeira e casca ( $\mathrm{M}_{\text {Ligmad }}$ e $\mathrm{M}_{\text {Ligcasca }}$ ) (Tabela 2) permitem constatar, para essas variáveis, que houve efeito significativo para os fatores clones e espaçamento, assim como para sua interação, evidenciando uma dependência entre os fatores (Tabela 3). O efeito da interação clone $\mathrm{x}$ espaçamento não foi significativo $(\mathrm{p}<0,05)$ para os teores de hemicelulose da madeira e casca e lignina da casca, mas na análise dos fatores principais evidenciou efeito significativo do espaçamento e de clone $(\mathrm{p}<0,05)$ para esses teores (Tabela 4). Para os teores de cinza, não foram observados efeitos significativos de clone e idade, assim como para a sua interação.

Tabela 2. Resumo da análise de variância para os teores de celulose, hemicelulose, lignina, cinzas da madeira e casca e de biomassa de holocelulose da madeira ( $\left.\mathbf{M}_{\text {holomad }}\right)$, biomassa de holocelulose da casca $\left(\mathrm{M}_{\text {holocasca }}\right)$, biomassa de lignina da madeira $\left(\mathrm{M}_{\text {Ligmad }}\right)$ e biomassa de lignina da casca $\left(\mathrm{M}_{\text {Ligcasca }}\right)$.

Table 2. Summary of the analysis of variance for the contents of cellulose, hemicellulose, lignin, wood ash and bark and wood holocellulose biomass $\left(\mathrm{M}_{\text {holomad }}\right)$, holocellulose husk biomass $\left(\mathrm{M}_{\text {holocasca }}\right)$, wood lignin biomass $\left(\mathrm{M}_{\text {Ligmad }}\right)$ and biomass Of shell lignin $\left(\mathrm{M}_{\text {Ligcasca }}\right)$.

\begin{tabular}{|c|c|c|c|c|c|c|c|c|c|c|c|c|c|}
\hline \multirow{3}{*}{$\begin{array}{l}\text { Fonte de } \\
\text { variação }\end{array}$} & \multirow{3}{*}{ GL } & \multicolumn{12}{|c|}{ Quadrado médio } \\
\hline & & \multicolumn{2}{|c|}{$\%$ Celulose } & \multicolumn{2}{|c|}{$\%$ Hemicelulose } & \multicolumn{2}{|c|}{$\%$ Lignina } & \multicolumn{2}{|c|}{$\%$ Cinzas } & \multirow[t]{2}{*}{$\mathbf{M}_{\text {holomad }}$} & \multirow[t]{2}{*}{$\mathbf{M}_{\text {holocasca }}$} & \multirow[t]{2}{*}{$\mathbf{M}_{\text {Ligmad }}$} & \multirow[t]{2}{*}{$\mathbf{M}_{\text {Ligcasca }}$} \\
\hline & & Madeira & Casca & Madeira & Casca & Madeira & Casca & Madeira & Casca & & & & \\
\hline Clone & 2 & $96,95^{*}$ & $26,86^{*}$ & $177,07 *$ & $92,98^{*}$ & $24,55^{*}$ & $56,61^{*}$ & $0,010^{\mathrm{ns}}$ & $0,004^{\mathrm{ns}}$ & $8,31^{*}$ & $0,195^{*}$ & $0,6612^{*}$ & $0,0163^{*}$ \\
\hline Espaçamento & 2 & $4,15^{\mathrm{ns}}$ & $9,07 *$ & $7,63^{*}$ & $2,08^{\mathrm{ns}}$ & $10,62^{*}$ & $9,32^{*}$ & $0,033^{\text {ns }}$ & $0,012^{\mathrm{ns}}$ & $13,29 *$ & $0,178^{*}$ & $0,3652^{*}$ & $0,0066^{*}$ \\
\hline $\begin{array}{c}\text { Clone* }^{*} \\
\text { Espaçamento }\end{array}$ & 4 & $5,07^{*}$ & $12,06^{*}$ & $2,27^{\mathrm{ns}}$ & $1,34^{\mathrm{ns}}$ & $2,55^{*}$ & $3,19^{\mathrm{ns}}$ & $0,023^{\text {ns }}$ & $0,019^{\mathrm{ns}}$ & $0,39 *$ & $0,028^{*}$ & $0,0152 *$ & $0,0013 *$ \\
\hline Erro & 27 & 1,50 & 1,06 & 1,25 & 2,28 & 0,65 & 1,73 & 0,009 & 0,007 & 0,12 & 0,002 & 0,0053 & 0,00021 \\
\hline Total & 35 & & & & & & & & & & & & \\
\hline
\end{tabular}

GL: graus de liberdade; *significativo ao nível de $5 \%$ de probabilidade $(\mathrm{p}<0,05)$; ${ }^{\text {ns }}$ não significativo ao nível de $5 \%$ de probabilidade $(\mathrm{p} \geq 0,05)$.

Analisando o efeito de clone dentro dos espaçamentos (Tabela 3), constatou-se que os clones 1 e 2 apresentaram médias semelhantes entre si e superiores ao clone 3 para as variáveis Ht, DAP, IMA, MSM, MSC e MST, exceto no espaçamento $3 \times 3 \mathrm{~m}$ onde o clone 2 apresentou médias significativamente superiores aos outros dois clones. Os maiores teores de celulose e de lignina na madeira foram observados nos clones 1 e 2 , que foram significativamente superiores ao clone 3. Quanto aos teores de celulose na casca, os clones 1 e 3 apresentaram médias significativamente superiores ao clone 2 no espaçamento $3 \times 3 \mathrm{~m}$, teores semelhantes no espaçamento $3 \times 1,5 \mathrm{~m}$, e no espaçamento 3x1 m o clone 1 apresentou as maiores médias em relação aos clones 2 e 3 .

Verificando o efeito do espaçamento dentro de cada clone, constatou-se para Ht diferença significativa somente para o clone 1 no espaçamento $3 \times 3 \mathrm{~m}$, onde foi observada média de Ht inferior aos outros dois 
espaçamentos. Para o DAP verificou-se que o espaçamento $3 \times 3 \mathrm{~m}$ proporcionou médias significativamente superiores para os três clones estudados. No espaçamento 3x1 m todos os clones apresentaram maior IMA, MSM, MSC e MST, em razão da maior densidade de árvores por unidade de área. Para os clones 1 e 3 os três espaçamentos proporcionaram médias semelhantes para o teor de celulose na madeira, e o clone 2 apresentou menor valor dessa variável no espaçamento $3 \times 1,5 \mathrm{~m}$. Quanto ao teor de celulose na casca, o espaçamento $3 \times 1 \mathrm{~m}$ apresentou valor médio significativamente superior para o clone 1, o clone 2 apresentou as menores médias no espaçamento $3 \times 3 \mathrm{~m}$, e os valores médios dessa variável foram semelhantes para o clone 3 nos três espaçamentos. Já para o teor de lignina na madeira, observaram-se para os clones 1 e 2 valores médios superiores no espaçamento $3 \times 3 \mathrm{~m}$, e para o clone 3 as médias foram semelhantes nos três espaçamentos testados.

Tabela 3. Valores médios e teste de comparação múltipla realizado para as características tecnológicas da madeira e casca que apresentaram interação entre os fatores clone e espaçamento.

Table 3. Mean values and multiple comparison test performed for the technological characteristics of the wood and bark that presented interaction between clone factors and spacing.

\begin{tabular}{|c|c|c|c|c|}
\hline \multirow{2}{*}{ Variáveis } & \multirow{2}{*}{ Clones } & \multicolumn{3}{|c|}{ Espaçamentos (m) } \\
\hline & & $\mathbf{3 , 0} \times \mathbf{3 , 0}$ & $3,0 \times 1,5$ & $3,0 \times 1,0$ \\
\hline \multirow{3}{*}{ Ht (m) } & 1 & $5,74 \mathrm{bB}$ & $6,26 \mathrm{aA}$ & $6,52 \mathrm{aA}$ \\
\hline & 2 & $6,65 \mathrm{aA}$ & $6,21 \mathrm{aA}$ & $6,21 \mathrm{aA}$ \\
\hline & 3 & $4,61 \mathrm{cA}$ & $4,85 \mathrm{bA}$ & $5,07 \mathrm{bA}$ \\
\hline \multirow{3}{*}{$\mathrm{DAP}(\mathrm{cm})$} & 1 & $5,25 \mathrm{bA}$ & $4,57 \mathrm{aB}$ & $4,21 \mathrm{aC}$ \\
\hline & 2 & $5,72 \mathrm{aA}$ & $4,72 \mathrm{aB}$ & $4,15 \mathrm{aC}$ \\
\hline & 3 & $3,50 \mathrm{cA}$ & $3,28 \mathrm{bB}$ & $3,18 \mathrm{bB}$ \\
\hline \multirow{3}{*}{$\operatorname{IMA}\left(\mathrm{m}^{3} \mathrm{ha}^{-1}\right)$} & 1 & $8,29 \mathrm{bC}$ & $13,65 \mathrm{aB}$ & $18,20 \mathrm{aA}$ \\
\hline & 2 & $11,65 \mathrm{aC}$ & $14,99 \mathrm{aB}$ & $17,89 \mathrm{aA}$ \\
\hline & 3 & $3,93 \mathrm{cC}$ & $6,05 \mathrm{bB}$ & $9,80 \mathrm{bA}$ \\
\hline \multirow{3}{*}{$\operatorname{MSC}\left(\mathrm{Mg} \mathrm{ha}^{-1}\right)$} & 1 & $0,58 \mathrm{bC}$ & $0,85 \mathrm{aB}$ & $1,15 \mathrm{aA}$ \\
\hline & 2 & $0,70 \mathrm{aB}$ & $0,83 \mathrm{aB}$ & $0,90 \mathrm{bA}$ \\
\hline & 3 & $0,31 \mathrm{bB}$ & $0,42 \mathrm{bB}$ & $0,61 \mathrm{cA}$ \\
\hline \multirow{3}{*}{$\operatorname{MSM}\left(\mathrm{Mg} \mathrm{ha}^{-1}\right)$} & 1 & $2,72 \mathrm{bC}$ & $4,62 \mathrm{aB}$ & $6,16 \mathrm{aA}$ \\
\hline & 2 & $3,65 \mathrm{aC}$ & $4,83 \mathrm{aB}$ & $5,70 \mathrm{aA}$ \\
\hline & 3 & $1,62 \mathrm{cC}$ & $2,44 \mathrm{bB}$ & $4,03 \mathrm{bA}$ \\
\hline \multirow{3}{*}{$\operatorname{MST}\left(\mathrm{Mg} \mathrm{ha}^{-1}\right)$} & 1 & $3,31 \mathrm{bC}$ & $5,47 \mathrm{aB}$ & $7,32 \mathrm{aA}$ \\
\hline & 2 & $4,35 \mathrm{aC}$ & $5,67 \mathrm{aB}$ & $6,61 \mathrm{aA}$ \\
\hline & 3 & $1,94 \mathrm{cC}$ & $2,86 \mathrm{bB}$ & $4,64 \mathrm{bA}$ \\
\hline \multirow{3}{*}{ Celulose madeira (\%) } & 1 & $59,64 \mathrm{aA}$ & $59,02 \mathrm{aA}$ & $61,49 \mathrm{aA}$ \\
\hline & 2 & $59,23 \mathrm{aAB}$ & $58,94 \mathrm{aB}$ & $61,78 \mathrm{aA}$ \\
\hline & 3 & $54,52 \mathrm{bA}$ & $55,04 \mathrm{bA}$ & $53,44 \mathrm{bA}$ \\
\hline \multirow{3}{*}{ Celulose casca $(\%)$} & 1 & $43,98 \mathrm{aB}$ & $42,88 \mathrm{aB}$ & $48,69 \mathrm{aA}$ \\
\hline & 2 & $40,84 \mathrm{bB}$ & $43,05 \mathrm{aA}$ & $41,35 \mathrm{cAB}$ \\
\hline & 3 & $43,29 \mathrm{aA}$ & $44,08 \mathrm{aA}$ & $43,97 \mathrm{bA}$ \\
\hline \multirow{3}{*}{ Lignina madeira (\%) } & 1 & $19,85 \mathrm{aA}$ & $17,13 \mathrm{aB}$ & $16,28 \mathrm{aB}$ \\
\hline & 2 & $18,90 \mathrm{aA}$ & $17,78 \mathrm{aB}$ & $16,33 \mathrm{aB}$ \\
\hline & 3 & $14,88 \mathrm{bA}$ & $15,18 \mathrm{bA}$ & $14,50 \mathrm{bA}$ \\
\hline
\end{tabular}

Ht: altura total; DAP: diâmetro a altura do peito; Vs/c: volume de madeira sem casca; Vcasca: volume de casca; Vc/c: volume de madeira com casca; MSC: biomassa seca da casca; MSM: biomassa seca da madeira; MST: biomassa seca total. Médias seguidas pela mesma letra minúscula na coluna e maiúscula na linha, não diferem entre si, ao nível de $5 \%$ de significância pelo teste de Tukey.

$\mathrm{Na}$ tabela 4 encontram-se os testes de comparação realizados, considerando os clones e espaçamentos como os fatores de variação, uma vez que a interação clone x espaçamento não foi significativa $(\mathrm{p}<0,05)$ para 0 percentual de casca, teor de cinzas da madeira e casca, teor de hemicelulose da madeira e casca, e teor de lignina da casca. Não foram observadas diferenças significativas do percentual de casca e dos teores de cinzas da madeira e da casca entre clones avaliados. Os maiores teores de hemicelulose da madeira e casca foram encontrados no clone 3, enquanto o clone 1 apresentou o menor teor de lignina da casca. Não foram constatadas diferenças significativas entre os espaçamentos para os teores de cinzas da madeira e casca e de hemicelulose da casca. No espaçamento $3 \times 3 \mathrm{~m}$ foi observado o maior percentual de casca e os menores teores de hemicelulose da madeira, e no espaçamento $3 \times 1 \mathrm{~m}$ constatou-se o menor teor de lignina da casca. 
Tabela 4. Efeito de clones e de espaçamento em algumas das caraterísticas tecnológicas madeira e casca.

Table 4. Effect of clones and spacing on some of the technological characteristics wood and bark.

\begin{tabular}{|c|c|c|c|c|c|c|}
\hline \multirow{2}{*}{ Variáveis } & \multicolumn{3}{|c|}{ Clones } & \multicolumn{3}{|c|}{ Espaçamentos (m) } \\
\hline & $\mathbf{1}$ & 2 & 3 & $3 \times 3$ & $3 \times 1,5$ & $3 \times 1$ \\
\hline$\%$ de casca & $20,38 \mathrm{a}$ & $20,58 \mathrm{a}$ & $21,84 \mathrm{a}$ & $22,39 \mathrm{a}$ & $20,67 \mathrm{~b}$ & $19,73 \mathrm{~b}$ \\
\hline$\%$ cinza madeira & $0,36 \mathrm{a}$ & $0,37 \mathrm{a}$ & $0,31 \mathrm{a}$ & $0,52 \mathrm{a}$ & $0,48 \mathrm{a}$ & $0,56 \mathrm{a}$ \\
\hline$\%$ cinza casca & $0,52 \mathrm{a}$ & $0,50 \mathrm{a}$ & $0,54 \mathrm{a}$ & $0,28 \mathrm{a}$ & $0,36 \mathrm{a}$ & $0,40 \mathrm{a}$ \\
\hline$\%$ hemicelulose madeira & $15,33 \mathrm{~b}$ & $16,53 \mathrm{~b}$ & $23,54 \mathrm{a}$ & $17,45 \mathrm{~b}$ & $19,24 \mathrm{a}$ & $18,71 \mathrm{ab}$ \\
\hline$\%$ hemicelulose casca & $17,31 \mathrm{~b}$ & $15,10 \mathrm{c}$ & $21,43 \mathrm{a}$ & $17,39 \mathrm{a}$ & $18,23 \mathrm{a}$ & $18,22 \mathrm{a}$ \\
\hline$\%$ lignina casca & $14,68 \mathrm{~b}$ & $19,30 \mathrm{a}$ & $18,68 \mathrm{a}$ & $18,63 \mathrm{a}$ & $17,41 \mathrm{ab}$ & $16,61 \mathrm{~b}$ \\
\hline
\end{tabular}

Médias seguidas pela mesma letra não diferem entre si ao nível de 5\% de significância pelo teste de Tukey.

As massas específicas básicas da madeira e casca dos diferentes clones não foram afetadas pelo espaçamento, houve somente diferenças significativas $(\mathrm{p}<0,05)$ entre os clones. Verifica-se pela figura 1 , que os maiores valores médios para massa específica básica da casca foram observados nos clones 1 e 3 , enquanto que o maior valor para massa específica básica da madeira foi observado no clone 3 .

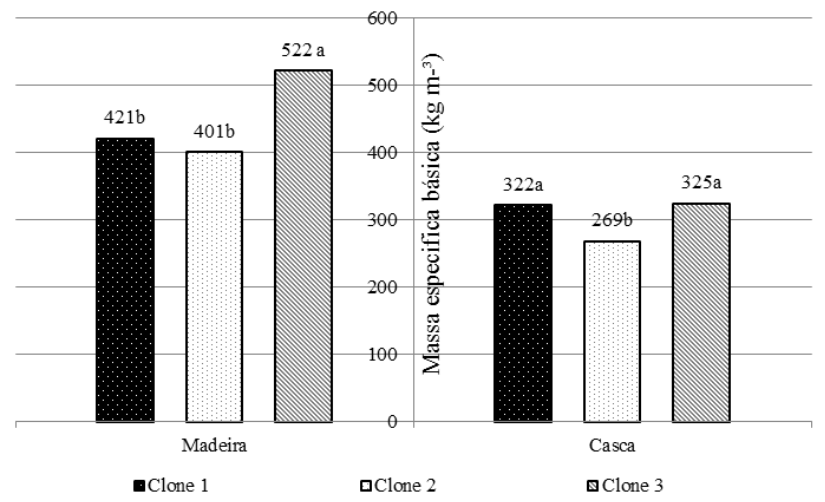

Figura 1. Massa específica da madeira e de casca $\left(\mathrm{kg} . \mathrm{m}^{-3}\right)$ em função dos clones aos 12 meses de idade. Médias seguidas pela mesma letra, não diferem entre si, ao nível de $5 \%$ de probabilidade, pelo teste de Tukey.

Figure 1. Specific wood and bark mass $\left(\mathrm{kg} . \mathrm{m}^{-3}\right)$ as a function of the clones at 12 months of age. Means followed by the same letter do not differ at the level of $5 \%$ probability by Tukey test.

Os três clones nos espaçamentos mais adensados obtiveram maior produção de biomassa de lignina tanto para madeira quanto para casca (Figura 2). Os clones 1 e 2 apresentaram produção de biomassa de lignina semelhantes nos espaçamentos $3 \times 1 \mathrm{~m}$ e no $3 \times 1,5 \mathrm{~m}$. Entretanto, no espaçamento $3 \times 3 \mathrm{~m}$, o clone 2 apresentou maior produção de biomassa de lignina da madeira em relação aos clones 1 e 3 .

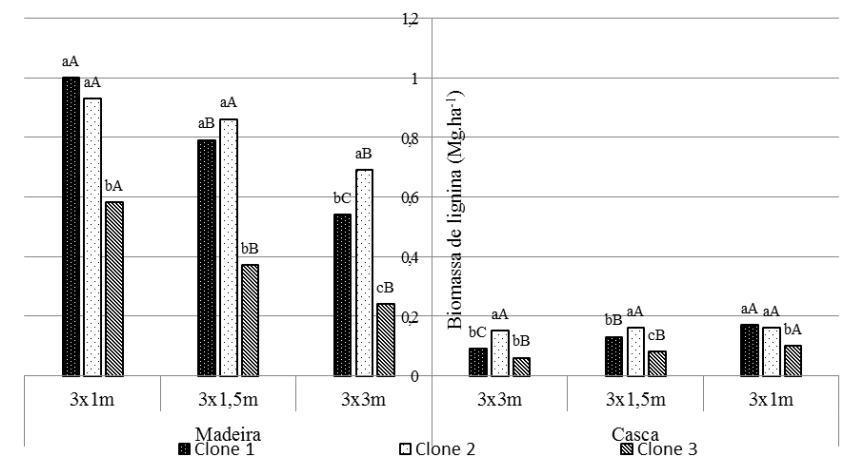

Figura 2. Biomassa de lignina $\left(\mathrm{Mg}_{\mathrm{h}} \mathrm{ha}^{-1}\right)$ de três clones aos 12 meses de idade sob três espaçamentos diferentes. Médias seguidas da mesma letra maiúscula entre os espaçamentos e minúscula entre clones não diferem entre si, ao nível de 5\% de significância, pelo teste de Tukey.

Figure 2. Lignin biomass $\left(\mathrm{Mg}_{\mathrm{g}} \mathrm{ha}^{-1}\right)$ of three clones for 12 months under three different spacings. Means followed by the same capital letter in the spacings between clones and lowercase do not differ at the $5 \%$ level of significance, by Tukey test. 
Para os três clones, o espaçamento mais adensado $(3 \mathrm{x} 1 \mathrm{~m})$ produziu maior biomassa de holocelulose para madeira e casca (Figura 3). Os clones 1 e 2 apresentaram produção de biomassa de holocelulose da madeira semelhantes nos espaçamentos $3 \times 1 \mathrm{~m}$ e no $3 \times 1,5 \mathrm{~m}$, e superiores ao clone 3 . No espaçamento $3 \times 3 \mathrm{~m}$, o clone 2 apresentou maior produção de holocelulose em relação aos clones 1 e 3 .

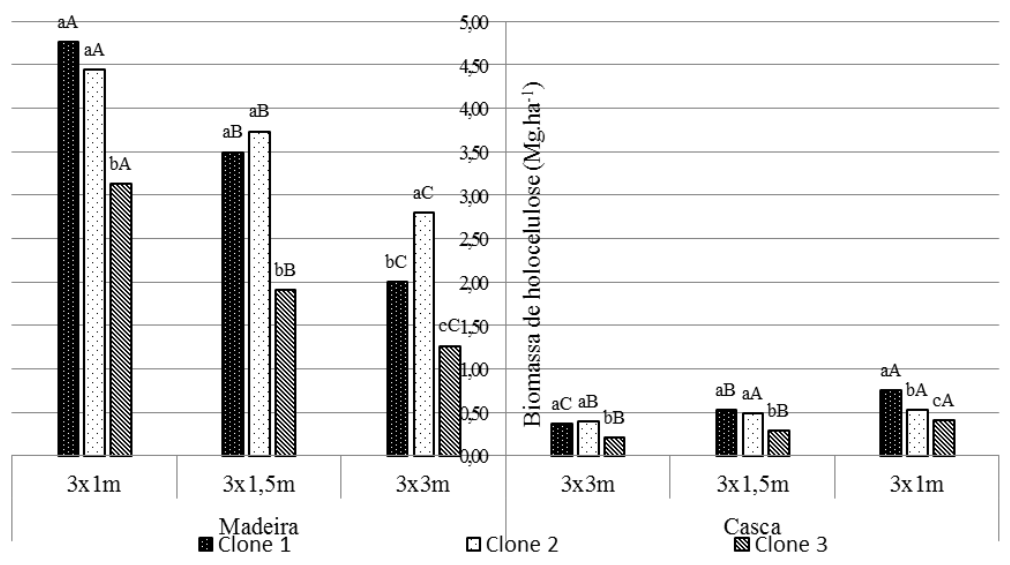

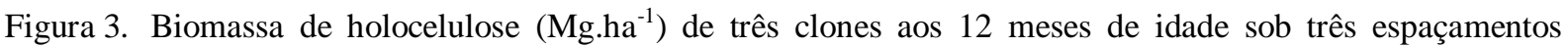
diferentes. Médias seguidas da mesma letra maiúscula entre os espaçamentos e minúscula entre clones não diferem entre si, ao nível de $5 \%$ de significância, pelo teste de Tukey.

Figure 3. Holocelulose biomass $\left(\mathrm{Mg} \cdot \mathrm{ha}^{-1}\right)$ of three clones for 12 months under three different spacings. Means followed by the same capital letter in the spacings between clones and lowercase do not differ at the $5 \%$ level of significance, by Tukey test.

\section{DISCUSSÃO}

\section{Avaliação do crescimento}

Os valor médio de altura total do clone 1 observado no espaçamento $3 \times 3 \mathrm{~m}$ foi inferior quando comparado com os valores médios obtidos nos espaçamentos 3x1,5 e 3x1 m. Já os clones 2 e 3 tiveram médias semelhantes de altura total em todos os espaçamentos testados. Pode-se inferir, para essa idade de avaliação, que não houve influência dos espaçamentos mais adensados na limitação do crescimento em altura das plantas. Leles et al. (2001) ao estudar árvores de Eucalyptus spp., aos 52 meses de idade, plantadas em diferentes espaçamentos, observaram pequenas diferenças entre os valores de altura total das plantas nos espaçamentos testados. Segundo esses mesmos autores, as diferenças em altura são bastante reduzidas quando comparadas às de diâmetro das plantas, que apresentam maiores valores em espaçamentos mais amplos.

Quanto ao DAP, pode-se inferir que o aumento do espaçamento entre as plantas influenciou para o maior incremento dessa variável. Assim, mesmo em povoamentos jovens, a concorrência entre as plantas em espaçamentos reduzidos pode afetar o crescimento em DAP. Ferreira et al. (2014), avaliando o crescimento em DAP de um clone híbrido de eucalipto conduzido em diferentes espaçamentos, verificaram que os espaçamentos maiores proporcionaram os maiores valores dessa variável. Segundo Stape et al. (2010), em espaçamentos mais adensados a proximidade dos sistemas radiculares aumenta sensivelmente a competição por água, afetando assim o crescimento diamétrico das plantas.

\section{Análise química da biomassa}

Os clones 1 e 2 apresentaram as maiores médias dos valores de celulose na madeira, enquanto o clone 3 apresentou as maiores médias do teor de hemicelulose na madeira e casca. A análise desses teores é imprescindível na avaliação dos materiais genéticos destinados à bioenergia, pois além de corresponder a uma fração significativa das biomassas, tanto a celulose quanto a hemicelulose, apresentam perfil instável e pouco resistente diante da degradação térmica da madeira (SANTOS et al., 2011).

Os teores de lignina encontrados na madeira ficaram entre $14,50 \%$ e $19,85 \%$, e os teores de lignina na casca variaram de $14,68 \%$ a $19,30 \%$. Os resultados obtidos para os teores de lignina podem ser considerados baixos quando comparados aos teores de celulose e hemicelulose na madeira e casca, sendo esses valores inferiores aos verificados em outros estudos. Moulin et al. (2015), estudando o efeito de diferentes espaçamentos na composição química da madeira de um clone hibrido de Eucalyptus grandis x E. urophylla, aos 12 meses de 
idade, observaram teores de lignina entre 27,8 a 32,2\%, sendo que os maiores teores de lignina foram obtidos nos maiores espaçamentos. Protásio et al. (2014) observaram teores de lignina entre 27,8 a 32,2\% em quatro clones de Eucalyptus, aos 57 e 69 meses de idade. Observou-se ainda, para os clones 2 e 3, o decréscimo dos teores de lignina na casca do maior espaçamento para os espaçamentos mais adensados, e igual tendência para o teor de lignina na madeira para os clones 1 e 2 . Zanuncio et al. (2013), avaliando a composição química da madeira de um clone híbrido de Eucalyptus grandis x E. urophylla constataram que tratamentos com maior espaçamento entre as plantas, favoreceram a biossíntese de compostos como ligninas e extrativos. Em razão da sua maior estabilidade térmica, o teor de lignina pode ser considerado uma das principais características da madeira e da casca associadas à produção de energia (GANI; NARUSE, 2007).

Os três clones estudados apresentaram baixa produção de cinzas, seja na madeira ou casca, situando esse parâmetro dentro da faixa observada em outros trabalhos para clones comerciais indicados para bioenergia (MOULIN et al., 2015; NEVES et al., 2013). Esses resultados são positivos, uma vez que os minerais presentes nas cinzas são indesejáveis na geração direta de bioenergia, as cinzas podem formar incrustações e corrosão em tubulações e equipamentos utilizados na produção de bioenergia (PROTÁSIO et al., 2014).

\section{Massa especifica básica}

Quanto à massa específica básica da casca e da madeira, como apenas o fator clone foi significativo, pode-se inferir que essa variável é uma propriedade mais influenciada pela herança genética do que pelos fatores ambientais, como sugere Trugilho et al. (2010). Eloy et al. (2014), ao avaliarem os valores de massa específica básica da madeira de Eucalyptus grandis, também verificaram que não houve variação significativa desse parâmetro em diferentes espaçamentos de plantio, aos 12 meses de idade. Segundo esses mesmos autores, a massa específica básica da espécie variou de 0,365 a $0,391 \mathrm{~g} . \mathrm{cm}^{-3}$, valores inferiores aos encontrados para todos os materiais genéticos testados no presente estudo.

Para Vale et al. (2002), ao se utilizar materiais genéticos destinados à produção direta de calor, que apresentem baixa massa específica básica, a queima será rápida, propiciando menor produção de energia por unidade de volume, ao contrário do que ocorre com materiais com maiores valores de massa específica básica. Para a produção de bioenergia, são recomendados genótipos com maiores valores de massa específica básica, uma vez que essa propriedade está diretamente relacionada à produção de biomassa seca, e consequentemente de energia (NEVES et al., 2013).

Os resultados encontrados no estudo sugerem que as madeiras mais densas possuem menores teores de lignina. Resultado semelhante foi observado por Trugilho et al. (1996), que verificaram que o teor de lignina apresentou correlação negativa com a densidade básica e com teor de holocelulose, indicando que, quanto menor for o teor de lignina, maior será o teor de holocelulose e, consequentemente, maior será a massa especifica básica.

\section{Biomassa seca da madeira e casca}

De maneira geral, observou-se uma relação direta da densidade de plantio com a produção de biomassa seca para os três clones avaliados. Dessa forma, nos espaçamentos com maior densidade de plantas, foram observados os maiores valores de biomassa total quando comparados com os espaçamentos com menor densidade de plantas. Caron et al. (2015) em estudo que visou determinar a capacidade de produção de biomassa da parte aérea de quatro espécies florestais, também verificaram que o aumento da densidade de plantas apresentou relação direta com a produção de biomassa por unidade de área, aos 12 e 36 meses. Resultados similares foram observados em plantios clonais de eucalipto por Berger et al. (2002) e Sereghetti et al. (2015).

Segundo Müller et al. (2005), deve-se ressaltar que, com o tempo, a quantidade de biomassa estocada num determinado sítio florestal tende a se igualar em diferentes espaçamentos de plantio. Segundo os mesmos autores, nos plantios mais adensados, a maior concentração de árvores por unidade de área impõe uma rápida estagnação do crescimento em idades mais jovens, ocorrendo o inverso em plantios menos adensados. Em razão disso, é necessário conhecer a dinâmica da produção de biomassa desde as fases iniciais de crescimento, de modo a definir as densidades de plantio e selecionar os materiais genéticos mais aptos à produção de bioenergia.

\section{Biomassa de lignina e holocelulose}

Os resultados encontrados no presente estudo permitem inferir que a maior produção de biomassa de lignina e de holocelulose foram obtidas no espaçamento $3 \times 1 \mathrm{~m}$, visto que nesse espaçamento ocorre uma maior produção de biomassa seca, tanto para madeira quanto para a casca. Os clones 1 e 2 apresentam produção de biomassa de lignina e holocelulose semelhantes, e superiores ao clone 3, nos espaçamentos 3x1 m e 3x1,5 m, e no espaçamento $3 \times 3 \mathrm{~m}$, o clone 2 apresentou produção significativamente superior aos demais clones. Esse fato relaciona-se em grande parte com a maior produção de biomassa seca desse clone no espaçamento mais aberto.

A estimativa da biomassa de lignina é fundamental para a seleção de materiais genéticos para o setor de 
bioenergia, haja vista que a lignina é o componente que sofre lento processo de degradação térmica, comparado aos outros constituintes primários da madeira (BOTREL et al., 2010). Já a biomassa de holocelulose de determinado material, é importante pode constituir em opção para utilização como outras fontes de energia, como a produção de bioetanol a partir dos açucares presentes na celulose e na hemicelulose.

Os resultados apresentados no presente estudo sugerem a necessidade de considerar as características dendrométricas, químicas e de massa especifica dos diferentes materiais genéticos de forma integrada, com o intuito de proceder à correta seleção da biomassa florestal destinada à produção de bioenergia. Para Santos et al. (2012) as pesquisas não devem buscar somente o incremento em volume, mas também na melhoria das características da biomassa florestal para cada uso a que se destina.

\section{CONCLUSÃO}

- A altura total dos clones não foi limitada pelos espaçamentos mais adensados, e o espaçamento $3 \times 3 \mathrm{~m}$ propiciou as maiores médias do DAP para os três clones.

- A massa específica básica da madeira e casca não sofreu influência dos espaçamentos. O clone 3 apresentou as maiores médias da massa específica básica.

- A produção de biomassa seca apresenta relação direta com a densidade de plantio, ou seja, quanto maior a densidade de plantio maior é a produção de biomassa por unidade de área.

- O espaçamento 3x1 m proporcionou a maior produção de biomassa seca para todos os clones estudados, sendo os clones 1 e 2 os mais indicados para o uso bioenergético por apresentarem os maiores valores de biomassa seca.

\section{REFERÊNCIAS}

ASSOCIAÇÃO BRASILEIRA DE NORMAS TÉCNICAS (ABNT) NBR 11941: madeira, determinação da densidade básica. Rio de Janeiro, 2003. 6 p.

BERGER, R.; SCHNEIDER, P. R.; FINGER, C. A. G.; HASELEI, C. R. Efeito do espaçamento e da adubação no crescimento de um clone de Eucalyptus saligna Ciência Florestal, Santa Maria, v. 12, n. 2, p. 75-87, 2002.

BOTREL, M. C. G.; TRUGILHO, P. F.; ROSADO, S. C. da S.; SILVA, J. R. M. Seleção de clones de Eucalyptus para biomassa florestal e qualidade da madeira. Scientia Forestalis, Piracicaba, v. 38, n. 86, p. 237-245, 2010.

CARNEIRO, A. de C. O.; CASTRO, A. F. N. M.; CASTRO, R. V. O.; SANTOS, R. C. dos; FERREIRA, L. P.; DAMÁSIO, R. A. P.; VITAL, B. R. Potencial energético da madeira de Eucalyptus sp. em função da idade e de diferentes materiais genéticos. Revista Árvore, Viçosa, v. 38, n. 2, p. 375-381, 2014.

CARON, B. O.; ELOY, E.; SOUZA, V. Q. de; SCHMIDT, D.; BALBINOT, R.; BEHLING, A.; MONTEIRO, G. C. Quantificação da Biomassa florestal em plantios de curta rotação com diferentes espaçamentos. Comunicata Scientiae, v. 6, n. 1, p. 106-112, 2015.

ELOY, E.; CARON, B. O.; SILVA, D. A.; SCHMIDT, D.; TREVISAN, R.; BEHLING, A.; ELLI, E. F. Influência do espaçamento nas características energéticas de espécies arbóreas em plantios de curta rotação. Revista Árvore, Viçosa, v. 38, n. 3, p. 551-559, 2014.

FERREIRA, D. H. A. A.; LELES, P. S. dos S.; MACHADO, E. C.; ABREU, A. H. M. de; ABILIO, F. M. Crescimento de clone de Eucalyptus urophylla x E. grandis em diferentes espaçamentos. Floresta, Curitiba, v. 44, n. 3, p. 431-440, 2014.

GANI, A.; NARUSE, I. Effect of cellulose and lignin content on pyrolysis and combustion characteristics for several types of biomass. Renewable Energy, Oxford, v. 32, n. 4, p. 649-661, 2007.

GUERRA, S. P. S.; SANCAS, K. P.; GARCIA, E. A.; SPINELLI, R. Eucalipto adensado: manejo para florestas energéticas. In: LEMOS, E.G. de M.; STADIOTTO, N. R. Bioenergia: Desenvolvimento, pesquisa e inovação. p. 125-161. São Paulo: Editora Cultura Acadêmica, 2012.

INSTITUTO ADOLFO LUTZ. Normas Analíticas do Instituto Adolfo Lutz. v.1: Métodos químicos e físicos para análise de alimentos, 4. ed. São Paulo: IMESP, 2008. 
LELES, P. S. S.; REIS, G. G.; REIS, M. G. F.; MORAIS, E. J. Crescimento, produção e alocação de matéria seca de Eucalyptus camaldulensis e E. pellita sob diferentes espaçamentos na região de cerrado, MG. Scientia Forestalis, Piracicaba, n. 59, p. 77-87, 2001.

MOULIN, J. C.; ARANTES, M. D. C.; VIDAURRE, G. B.; PAES, J. B.; CARNEIRO, A. de C. O. Efeito do espaçamento, da idade e da irrigação nos Componentes químicos da madeira de eucalipto Revista Árvore, Viçosa, v. 39, n. 1, p. 199-208, 2015.

MÜLLER, M. D.; COUTO, L.; LEITE, H. G.; BRITO, J. O. Avaliação de um clone de eucalipto estabelecido em diferentes densidades de plantio para produção de biomassa e energia. Biomassa \& Energia, v. 2, n. 3, p. 177$186,2005$.

NEVES, T. A.; PROTÁSIO, T. de P.; TRUGILHO, P. F.; VALLE, M. L. A.; SOUSA, L. C. de; VIEIRA, C. M. M. Qualidade da madeira de clones de Eucalyptus em diferentes idades para a produção de bioenergia. Revista de Ciências Agrárias, v. 56, n. 2, p. 139-148, 2013.

PROTÁSIO, T. de P.; NEVES, T. A.; REIS, A. A. dos; TRUGILHO, P. F. Efeito da idade e clone na qualidade da madeira de Eucalyptus spp visando à produção de bioenergia. Ciência Florestal, Santa Maria, v. 24, n. 2, p. 465477, 2014.

SANTOS, R. C. dos; CARNEIRO, A. de C. O.; CASTRO, A. F. M.; CASTRO, R. V. O.; BIANCHE, J. J.; SOUZA, M. M. de; CARDOSO, M. T. Correlações entre os parâmetros de qualidade da madeira e do carvão vegetal de clones de eucalipto. Scientia Forestalis, Piracicaba, n. 90, p. 221-230, 2011.

SANTOS, L. C.; CARVALHO, A. M. M. L.; PEREIRA, B. L. C.; OLIVEIRA, A. C.; CARNEIRO, A. C. O.; TRUGILHO, P. F. Propriedades da madeira e estimativas de massa, carbono e energia de clones de Eucalyptus plantados em diferentes locais. Revista Árvore, Viçosa, v. 36, n. 5, p. 971-980, 2012.

SEREGHETTI, G. C.; LANÇAS, K. P.; SARTORI, M. S.; REZENDE, M. A.; SOLER, R. R. Efeito do espaçamento no crescimento e na densidade básica da madeira de Eucalyptus urophylla x Eucalyptus grandis em florestas de ciclo curto. Energia na Agricultura, Botucatu, v. 30, n. 3, p. 257-262, 2015.

STAPE, J. L.; BINKLEY, D.; RYAN, M. G.; FONSECA, R. A.; LOOS; R. A. The Brazil eucalyptus potential productivity project: influence of water, nutrients and stand uniformity on wood production. Forest Ecology and Management, Amsterdan, v. 259, p. 1684-1694, 2010.

TRUGILHO, P. F.; LIMA, J. T.; MENDES, L. M. Influencia da idade nas características físico-químicas e anatômicas da madeira de Eucalyptus saligna. CERNE, Lavras, v. 2, n. 1, p. 94-111, 1996.

TRUGILHO, P. F.; ARANTES, M. D. C.; PÁDUA, F. A. de; ALMADO, R. P.; BALIZA, A. E. R. R. Estimativa de carbono fixado na madeira de um clone híbrido de Eucalyptus urophylla e Eucalyptus grandis. Cerne, Lavras, v. 16, p. 33-40, 2010.

VALE, A. T.; BRASIL, M. A. M.; LEÃO, A. L. Qualificação e caracterização energética da madeira e casca de espécies do cerrado. Ciência Florestal, Santa Maria, v. 12, n. 1, p. 71-80, 2002.

VAN SOEST, P. J. Use of detergents in the analysis of fibrous feeds II. A rapid method of the determination of fiber and lignin. J. Assoc. Official Agr. Chem. v. 26, n. 5, p. 829-835. 1970.

WASHUSEN, R.; BAKER, T.; MENZ, D.; MORROW, A. Effect of thinning and fertilizer on the cellulose crystallite width of Eucalyptus globulus. Wood Science and Technology, v. 39, n. 7, p. 569-578, 2005.

ZANUNCIO, A. J. V.; COLODETTE, J. L.; GOMES, F. J. B.; CARNEIRO, A. de C. O.; VITAL, B. R. Composição química da madeira de eucalipto com diferentes níveis de desbaste. Ciência Florestal, Santa Maria, v. 23, n. 4, p. 755-760, 2013. 